Original article

\title{
Seroprevalence of Toxocariasis in schoolchildren in Bushehr, Iran
}

\author{
Moradali Fouladvand ${ }^{\mathrm{a}}$, Afshin Barazesh ${ }^{\mathrm{b}, *}$ \\ ${ }^{a}$ The Persian Gulf Marine Biotechnology Research Center, The Persian Gulf Biomedical Sciences Research Institute, Bushehr University of Medical Sciences, Bushehr, Iran \\ ${ }^{\mathrm{b}}$ Department of Microbiology and Parasitology, Faculty of Medicine, Bushehr University of Medical Sciences, Bushehr, Iran
}

A R T I C L E IN F O

Keywords:

Toxocariasis

Primary school children

Bushehr

Iran

\begin{abstract}
A B S T R A C T
Background: Toxocariasis is an infection caused by larvae of a group of animal nematodes named Toxocara in human tissues. The disease often affects children under the age of 10 years. In this research, we decided to determine the prevalence of infection in schoolchildren of Bushehr city as a first study in the region. Methods: In this cross-sectional study, the samples were selected randomly among Bushehr primary school students between September 2011 and June 2012. ELISA method was used to measure anti-Toxocara canis IgG antibodies. Data were collected by questionnaire and analyzed using SPSS 18.0 software and Chi square test. Results: Of the 400 students studied, 48 (12\%) were positive for anti-Toxocara IgG antibodies. Results showed that there is no a statistically significant relationship between infection and some of the analyzed variables such as sex, abdominal pain, anorexia, chronic cough, diffuse pain, itching and skin sensitivity. However, with the habit of playing with soil, this relationship was significant.

Conclusion: Considering the importance of the disease in children and influence of harmful effects on their health, it is suggested that the methods for diagnosis be trained for clinicians in the area and periodically tests as well as teaching the prevention and control strategies should be considered to the educational staff and parents.
\end{abstract}

\section{Introduction}

Various nematodes such as Toxocara spp., Baylisascaris procyonis, Ascaris suum, Capillaria hepatica, etc. have the potential to cause visceral larva migrans (VLM) in humans, especially in children. Toxocariasis is an infection causing by larvae of three species of Toxocara spp. (T.canis, T.cati, T.leonina) in human tissues, which among all these agents, the second stage larvae of Toxocara canis is the main cause of the disease in all over the world.

Toxocara canis is commonly found in the intestine of dogs. The infection in dogs is usually mild, but if humans, as paratenic hosts, become infected by swallowing accidentally and ingesting infective eggs of these worms in contaminated soil, after hatching, the larvae penetrate the intestinal wall and migrate into the liver, brain, eyes and other tissues of the body causing toxocariasis. ${ }^{1-3}$ Since the larvae do not undergo any further development in these sites, they can cause several local reactions that are the basis of toxocariasis.

In most cases, Toxocara infections are not serious, and many people, especially adults infected by a small number of larvae (immature worms), may not notice any symptoms. The most severe cases are rare, but are more likely to occur in young children, who often play in dirt, or eat dirt contaminated by dog stool.
In general, three clinical forms of human toxocariasis have been identified: Visceral Larva Migrans (VLM), Ocular Larva Migrans (OLM) and Covert Toxocariasis (CT). Recently, another type is also known as Neurological Toxocariasis (NT). ${ }^{4-6}$ In most cases, there are no clinical symptoms except high eosinophilia. ${ }^{7,8}$ In VLM, the symptoms appear as fever, cough, abdominal pain, hepatomegaly and splenomegaly, anorexia and enlargement of the lymph nodes. ${ }^{9,10}$ In OLM, the migration of larvae into the eyes can cause granulomatous lesions in the retina, which is associated with loss of vision, strabismus and sometimes blindness. ${ }^{1,10}$ Toxocariasis affects most children under 12 years. ${ }^{11}$

Serological studies in different countries have shown a global distribution of toxocariasis and in some developed countries, it aligned with the prevalence of Enterobius vermicularis. There are many documented reports of toxocariasis in Iran. According to a study conducted by ELISA in 2002 on children under 10 years in Hamedan, west of Iran, the prevalence was $5.3 \%{ }^{6}$ and in another study carried out by the same method in Shiraz, southwest of Iran, the prevalence was $25.6 \%{ }^{12}$ Serological study on children aged 2-12 years old in Kermanshah province, west of Iran, had a prevalence of $8.4 \% .{ }^{10}$

Therefore, due to different and non-specific clinical features of toxocariasis, as well as the absence of any statistics on its prevalence in our region, we decided to determine the prevalence of infection using

\footnotetext{
* Corresponding author. Bushehr University of Medical Sciences, P. O. Box. 3631, Bushehr, Iran.

E-mail address: abarazesh@bpums.ac.ir (A. Barazesh).
} 
the sensitive and specific ELISA method as the first study in Bushehr, Iran in primary school children under 10 years and pave the way for further studies, especially in the field of prevention and control the disease.

\section{Materials and methods}

\subsection{Ethical approval and informed consent}

In this cross-sectional study, after coordination with the local education department, our license to attending at primary schools was issued by the security department of education. This study was approved by the ethics committee of the Bushehr University of Medical Sciences (Ethics committee code: 1388.26982). The samples were selected randomly among Bushehr primary school students between September 2011 and June 2012. An informed consent was signed by each of the parents of the participants.

\subsection{Sample size}

Various seroepidemiological studies of toxocariasis in children under ten years old in different parts of Iran showed a wide range of prevalence from $2 \%$ to around $25 \%$ and the highest prevalence rate (25.6\%) has been reported from Shiraz city. ${ }^{12}$ Since Shiraz city (Fars province) is the neighboring province of Bushehr and it's ecological conditions is somewhat similar to Bushehr, for calculating the sample size, we considered $20 \%$ as the baseline prevalence rate, the confidence interval of $95 \%$ and absolute error of $4 \%$. According to these considerations, sample size was calculated as 384 persons, but to be more confident, sample size was considered as 400 persons.

\subsection{Sample preparation}

Four hundred students were selected from all 20 primary schools in Bushehr city as randomly from different areas of the city and at a meeting organized for the parents of the students in coordination with primary schools managers, a questionnaire was completed for each student to collect demographic data and some of the variables involved in the prevalence.

\subsection{Experimental}

Blood samples were collected from the school students and transferred to the laboratory. After centrifugation at $2000 \mathrm{rpm}$ for $10 \mathrm{~min}$, separated sera were stored in $-70{ }^{\circ} \mathrm{C}$ until the test was performed. Sera were tested by anti-Toxocara canis IgG ELISA kit (IBL, Germany with sensitivity and specificity $>$ 95\%) and using ELISA reader (Biotek, USA). This Toxocara canis IgG ELISA kit is intended for the qualitative determination of antibodies against Toxocara canis circulating in human serum or plasma.

\subsection{Statistical analysis}

The results and data of the questionnaires, were analyzed using SPSS software version 18 and chi-square test and P.values. At levels $<0.03$, the $P$.values were considered as statistically significant.

\section{Results}

Among 400 students studied in five levels, 48 (12\%) were positive for anti-Toxocara IgG antibodies and of which, 19 (4.8\%) were boys and $29(7.2 \%)$ were girls. Results showed that there is no a statistically significant relationship between infection and sex (Table 1).

Other variables analyzed in this study, were abdominal pain, anorexia, chronic cough, diffuse pain, itching and skin sensitivity. The habit of playing with soil, was the only variable which it's relationship
Table 1

Distribution of anti-Toxocara canis IgG antibody in relation to some related variables.

\begin{tabular}{|c|c|c|c|c|c|}
\hline \multirow[t]{2}{*}{ Variable } & \multicolumn{2}{|c|}{ Positive } & \multicolumn{2}{|c|}{ Negative } & \multirow[t]{2}{*}{ P.value } \\
\hline & $n$ & $\%$ & $n$ & $\%$ & \\
\hline \multicolumn{6}{|l|}{ Sex } \\
\hline Female & 29 & 7.2 & 186 & 46.5 & $0.20 *$ \\
\hline Male & 19 & 4.8 & 166 & 41.5 & \\
\hline \multicolumn{6}{|c|}{ Abdominal pain } \\
\hline Yes & 23 & 5.7 & 178 & 44.5 & $0.42 *$ \\
\hline No & 25 & 6.3 & 174 & 43.5 & \\
\hline \multicolumn{6}{|l|}{ Anorexia } \\
\hline Yes & 11 & 2.8 & 106 & 26.5 & $0.19 *$ \\
\hline No & 37 & 9.2 & 246 & 61.5 & \\
\hline \multicolumn{6}{|c|}{ Chronic cough } \\
\hline Yes & 19 & 4.7 & 163 & 40.8 & $0.23 *$ \\
\hline No & 29 & 7.3 & 189 & 47.2 & \\
\hline \multicolumn{6}{|c|}{ Diffuse pain } \\
\hline Yes & 7 & 1.8 & 51 & 12.8 & $0.56^{*}$ \\
\hline No & 41 & 10.2 & 301 & 75.2 & \\
\hline \multicolumn{6}{|c|}{ Playing with soil } \\
\hline Yes & 17 & 4.2 & 72 & 18 & $0.02 *$ \\
\hline No & 31 & 7.8 & 280 & 70 & \\
\hline \multicolumn{6}{|l|}{ Itch } \\
\hline Yes & 3 & 0.8 & 25 & 6.3 & $0.255^{*}$ \\
\hline No & 45 & 11.2 & 327 & 81.7 & \\
\hline
\end{tabular}

$* P \leq 0.03$ level.

was statistically significant $(P<0.03)$ (Table 1$)$.

\section{Discussion}

Serological investigations have shown various results regarding the toxocariasis prevalence in different regions of Iran and also in different countries, which it depends on the study group, age, as well as the socio-cultural level of individuals. In the present study, the prevalence of toxocariasis in schoolchildren in Bushehr city was found to be about $12 \%$. In a study on primary school children in Shiraz, the prevalence of anti-Toxocara IgG antibodies has been reported $25.6 \%{ }^{12}$ Similar studies in Ahwaz, Hamedan and Kermanshah, southwest and west of Iran, have declared the prevalence of $2 \%, 5.3 \%$ and $8.46 \%$, respectively. ${ }^{3,6,10}$ Similar studies conducted in different parts of the world, have also achieved different results. In the studies on school age children in Turkey, Sri Lanka and Trinidad, the prevalence have been achieved $12.95 \%$, 43\%, and $62.3 \%$, respectively. ${ }^{11,13,14}$

In a Sero-epidemiological study conducted on school-aged children in eastern Taiwan by Chia-Kwung Fan and colleagues, 252 (76.6\%) of the 329 children had anti-Toxocara antibodies, which is very noticeable in comparison with similar studies. Using different cutt-off titres has been mentioned as a reason for not matching their study results with other similar studies. ${ }^{15}$

Differences in prevalence in different studies could be duo to some factors such as geographical location and ecological zone, as well as economic, social and cultural inhabitants ${ }^{8}$ and the high prevalence in some areas, can indicate the presence of infected dogs and cats in public places and high contamination of the soils in these areas with Toxocara eggs.

In this study there was no significant correlation between the prevalence of infection and gender of children studied, although it was expected that the prevalence be higher in boys, because of the high interest of boys in playing soil in comparison with girls. ${ }^{1,13,16}$ This finding is similar to some researches done in this field. ${ }^{3,12,15}$

Other variables analyzed in this study, were abdominal pain, anorexia, chronic cough, diffuse pain, itching and skin sensitivity. The habit of playing with soil, was the only variable which it's relationship was statistically significant $(P<0.03)$. This result is consistent with a study which has been done in eastern Taiwan among mountain 
aboriginal schoolchildren living in contaminated districts. ${ }^{15}$

Besides toxocariasis, there are other similar nematodes that have the same transmission structure and have a high zoonotic importance (Soiltransmitted nematodes), so in this regard, health education has a very important role in preventing the infection. Therefore, the risk of close contact with the dog, the role of dogs and cats in environmental contamination and increasing the chance of getting the infection should be indicated to the child's parents by government and health authorities. Moreover, sand dishes are used in children's parks must be protected with covers and the feces of dogs and cats in the children's playground, should be collected and buried. Finally, dogs and cats should be treated periodically with pyprazine or levamisole.

One of the strengths of the present study is the selection of schools with a large number from different areas as well as with a wide distribution in the city which can have a positive impact on the accuracy of the study and makes the prevalence rate more reliable. However, besides assessing the prevalence of anti-Toxocara antibodies in primary school children in Bushehr, it was better that soils of public places and city parks were also monitored for Toxocara eggs contamination. In such circumstances, the interpretation of the results and conclusions for controlling the disease status in the region were carefully considered. This can be considered as one of the limitations of this study.

\section{Conclusion}

Overall, $12 \%$ of the population studied in this research had antiTaxocara canis IgG antibodies that is noticeable and is almost similar with other regions in Iran. Therefore, considering the importance of the disease in children and influence of harmful effects on their health, it is suggested that the methods for diagnosis be trained for clinicians in the area and periodically tests as well as teaching the prevention and control strategies should be considered to the educational staff and parents. It should be prevented from playing children with puppies or cats aged between 3 and 6 weeks. Parasite eggs can stick to the skin of these animals and remain for a long time, so, after hugging or touching dogs and cats, hands should be washed well.

\section{Declaration of competing interest}

Authors declare that they have no conflict of interest.

\section{Acknowledgments}

This research is a project approved and financially supported by the Vice-Chancellor for Research of Bushehr University of Medical Sciences, Bushehr, Iran (Grant No. 1388.26982). Therefore, the authors are grateful to financial support of this plan.

\section{References}

1. Overgaauw PAM. General introduction aspects of toxocara epidemiology: human toxocarosis. Crit Rev Microbiol. 1997;23:215-231.

2. Gillespie S, Pearson RD. Principle and Practice of Clinical Parasitology. first ed. Virginia: Wiley \& Sons; 2001:501-509.

3. Alavi SM, Hosseini SA, Rahdar M, Salmanzadeh Sh, Nikkhuy Ar. Determination of seroprevalence rate of Toxocara canis in 6-15 years aged rural and urban school children in Ahvaz, Iran. Sci Med J. 2011;10(3):239-248.

4. Holland C, O'Connor P, Taylor MR, Hughes G, Girdwood RW, Smith H. Families, parks, gardens and toxocariasis. Scand J Infect Dis. 1991;23:225-231.

5. Fenoy S, Cuellar G, Guillen JL. Seroprevalence of toxocarisis in children and adults in Madrid and Tenerife, Spain. J Helminthol. 1996;70:109-113.

6. Fallah M, Azimi A, Taherkhani H. Seroprevalence of toxocariasis in children aged 1-9 years in Hamadan - 2002. ZUMSJ. 2005;13(50):44-50.

7. Kincekova J, Reiterova K, Dubinsky P. Larval toxocariasis and its clinical manifestation in childhood in the Slovak Republic. J Helminthol. 1999;73:323-328.

8. Taylor MR. The epidemiclogy of ocular toxocariasusis. $J$ Helminthol. 2001;75(2):109-118

9. Maclean JD, Graeme-Cook FM. Case 12-2002-A 50-year-old man with eosinophilia and fluctuating hepatic lesions. N Engl J Med. 2002;346(16):1232-1239.

10. Akhlaghi L, Ourmazdi H, Sarafnia A, Vaziri S, Jadidian K, Leghaii Z. An investigation on the toxocariasis seroprevalence in children (2-12 years old) from Mahidasht area of Kermanshah province (2003-2004). Iran Univ Med Sci. 2006;13(52):41-48.

11. Dogan N, Dinleyici EC, Bor O, Ozensoy Toz S, Ozbel Y. Seroepidemiological survey for Toxocara canis infection in the northwestern part of Turkey. Turk Parazitoloji Derg. 2007;31(4):288-291.

12. Sadjjadi SM, Khosravi M, Mehrabani D, Oryan A. Seroprevalence of Toxocara infection in school children in Shiraz, southern Iran. J Trop Pediatr. 2000;46:327-330.

13. Baboolal Sh, Rawlins SC. Seroprevalence of toxocariasis in schoolchildren in Trinidad. Trans R Soc Trop Med Hyg. 2002;96:139-143.

14. Iddawela DR, Kumarasir PVR, Wijesundera MDS. A Seroepidemiological study of Toxocariasis and risk factors for infection in children in Sri Lanka. Southeast Asian J Trop Med Publ Health. 2003;3(1):7-15.

15. Fan Chia-Kwung, Hung Chien-Ching, Du Wen-Yuan, Liao Chien-Wei, Su Kua-Eyre. Seroepidemiology of Toxocara canis infection among mountain aboriginal schoolchildren living in contaminated districts in eastern Taiwan. Trop Med Int Health. 2004;9(12):1312-1318.

16. Holland CV, O'Lorcain P, Taylor MR, Kelly A. Seroepidemiology of toxocariasis in school children. Parasitology. 1995;110:535-545. 